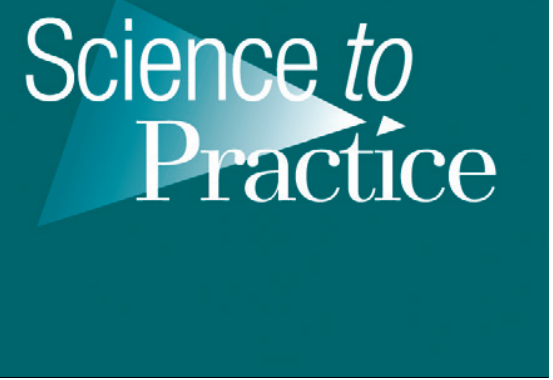

Peter L. Choyke, MD

Molecular Imaging Program

National Cancer Institute

10 Center Dr, MSC 1182 Bldg 10, Room B3B69F Bethesda, MD 20892

pchoyke@nih.gov

See page 746

\title{
Science to Practice: Monitoring
}

\section{Oncolytic Virus Therapy with Chemical} Exchange Saturation Transfer MR Imaging - Wishful Thinking?

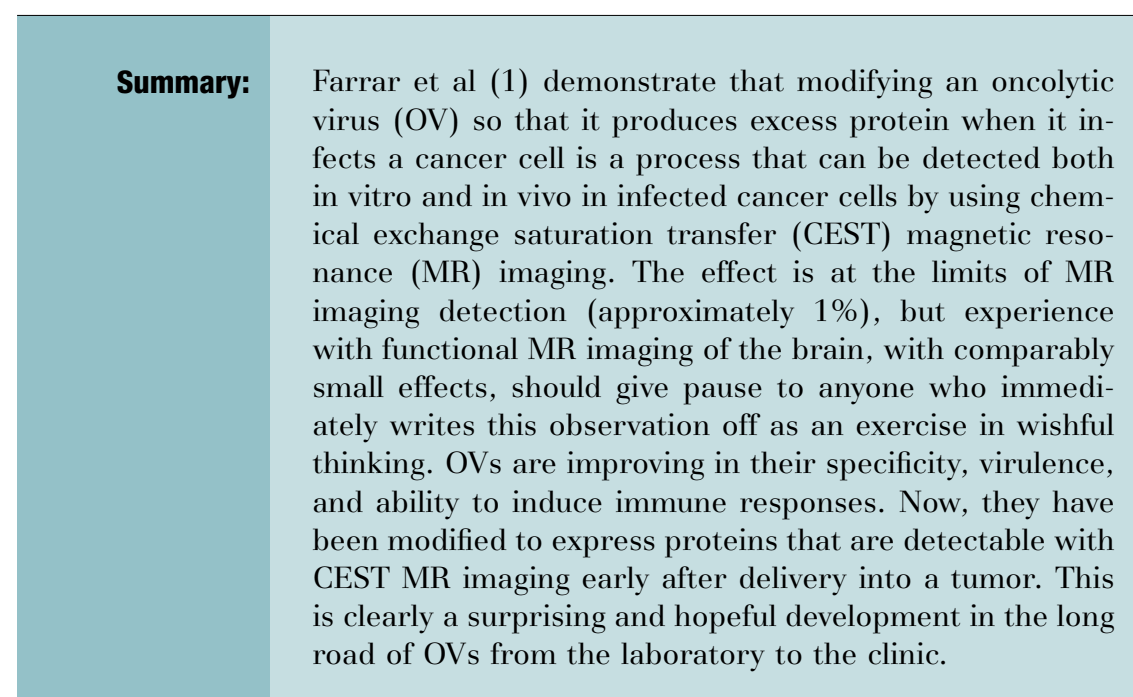

\section{The Setting}

OV therapy represents a new form of cancer treatment in which a replicating virus is injected, either systemically or intratumorally, and selectively enters cancer cells, replicates-thereby killing them-and then induces an immune reaction capable of killing noninfected cancer cells (2). Numerous trials of OVs

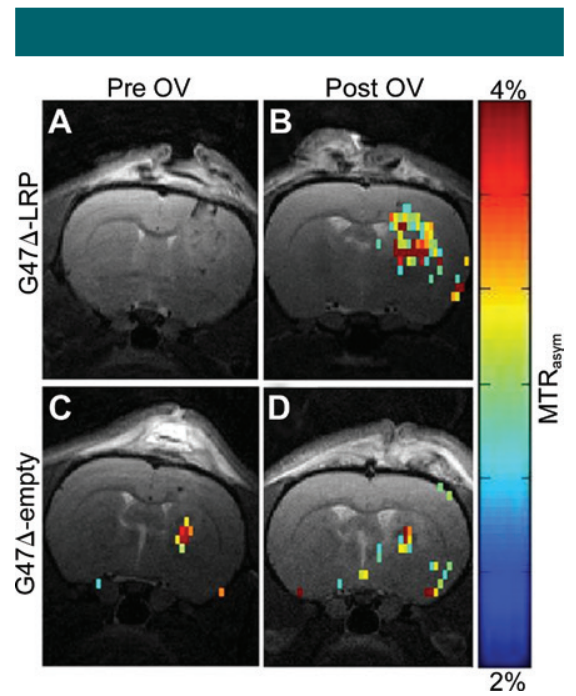

involving a broad range of highly lethal cancers are underway (3). Since the 1990 s, steady progress has been made in the cancer specificity and lethality of OVs, although there is yet to be a clinical breakthrough. Problems arise from the inability to deliver OVs, owing to permeability limitations, heterogeneous intratumoral distribution, and variable intratumoral replication once within the cell, among others. Nonetheless, OV therapy is considered part of the future of "precision medicine."

\section{The Science}

One recurrent problem with OV therapy is that it can be difficult to tell how much of the tumor has been infected and whether a "viral threshold" has been reached (2). Clearly, OV therapy could be improved if delivery could be assessed earlier. In this month's Radiology, Farrar et al describe the use of CEST MR imaging to detect the location of an OV designed to treat glioblastoma multiforme in a rodent model (1). The theory behind CEST imaging is that there are two pools of protons in the 
body, bulk ("free") water protons (from which the MR imaging signal is derived) and molecule-bound protons, which are typically bound to proteins. Bound protons are "off-resonant" with respect to bulk water protons, so that off-resonance irradiation (saturation) leads to loss of MR imaging signal intensity once the irradiated bound protons exchange with the bulk water. The effect is small, no more than 5\% under ideal circumstances, and relatively insensitive, typically requiring millimolar concentrations of protein to be detectable, but it is real $(4,5)$. By engineering the OV to produce lysine-rich proteins, CEST imaging can be used to detect the presence of the replicating OV within 8-10 hours after injection (Fig 1). This is not simply labeling the OV but rather a measurement of the product of the OV once it starts replicating. Thus, CEST MR imaging is feasible for determining if an $\mathrm{OV}$ is within the tumor and whether it is beginning the process of replication.

\section{The Practice}

The authors are quick to point out that there are limitations to the clinical applicability of this method. The laboratory provides idealized conditions, including high sensitivity (a 9.4-T magnet and dedicated hardware), lack of motion (anesthetized animals), and customized cell lines (expressing herpes thymidine kinase receptors designed for the OV), among other conditions that are not likely to be found in the clinical environment. Even under these ideal conditions, the CEST signal intensity change due to lysine-rich protein production by the OV is only about $1.5 \%$, which may be within the hourly variation of many clinical MR imaging units (definitely ours). Nonetheless, the principle of using a simple protein as a marker for OV delivery without additional exogenous injections is provocative. Many skeptics thought functional MR imaging for functional brain imaging, with its paltry $2 \%-5 \%$ change in signal intensity on MR images, was unlikely to be useful-and now it is a routine test in the neurosciences. However, this is not to minimize the challenges ahead. The authors point out that CEST MR imaging may only be valid early after OV delivery before cytotoxic effects have begun, which could independently alter CEST signal intensity. Nevertheless, it could be quite useful for determining the "functional" delivery of OVs to a tumor early on in therapy. The value of this is underscored by noting that only $40 \%$ of the tumor volume was exposed to OV in this study. Of course, at this point, CEST MR imaging is nonquantitative, and it is unclear how much signal intensity is needed to indicate that the viral threshold has been reached (2). Iterative CEST imaging during OV delivery could ensure more complete tumor exposure and presumably better effectiveness, but this link needs to be shown. Of course, these matters are largely technical, and the big questions about dose, safety, and effectiveness of OVs will dominate the direction of research in the coming years.

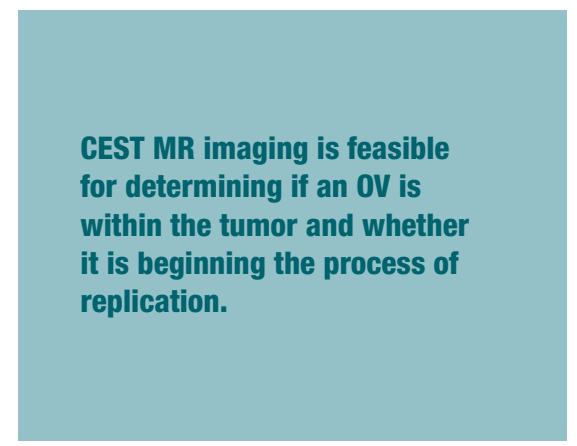

How does CEST MR imaging compare with reporter gene positron emission tomographic (PET) imaging? The latter requires the injection of a radioactive reporter that is entrapped within infected cells. Of course, such reporters are often taken up nonspecifically, leading to some uncertainty about the contribution of the OV to the uptake. PET imaging also requires ionizing radiation and the expense of producing another independent imaging agent, with its requisite chemical and regulatory hurdles. However, PET reporter gene imaging is also better for the detection of OV activity later on after delivery, since it is less likely to be affected by background changes in the tissue. Thus, CEST MR imaging and PET reporter imaging are likely to be complementary, with CEST imaging playing a greater role early in therapy and PET playing a greater role later.

Disclosures of Conflicts of Interest: P.L.C. disclosed no relevant relationships.

\section{References}

1. Farrar CT, Buhrman JS, Liu G, et al. Establishing the lysine-rich protein CEST reporter gene as a CEST MR imaging detector for oncolytic virotherapy. Radiology 2015;275(3): 746-754.

2. Russell SJ, Peng KW, Bell JC. Oncolytic virotherapy. Nat Biotechnol 2012;30(7): $658-670$.

3. Lichty BD, Breitbach CJ, Stojdl DF, Bell JC. Going viral with cancer immunotherapy. Nat Rev Cancer 2014;14(8):559-567.

4. Ahrens ET, Bulte JW. Tracking immune cells in vivo using magnetic resonance imaging. Nat Rev Immunol 2013;13(10):755-763.

5. Hingorani DV, Bernstein AS, Pagel MD. A review of responsive MRI contrast agents: 2005-2014. Contrast Media Mol Imaging 2014 Oct 29. [Epub ahead of print] 